\title{
Causal mechanisms in the clinical course and treatment of back pain
}

Lee $\mathrm{H},{ }^{1,2,3,4}$ Mansell G, ${ }^{5}$ McAuley JH, ${ }^{2,3}$ Kamper SJ, ${ }^{4,6}$ Hübscher $M,{ }^{2}$ Moseley GL, ${ }^{7}$ Wolfenden L, ${ }^{1,8}$ Hodder $\mathrm{R}^{1,8}$ Williams $\mathrm{C}^{1,4,6}$

1. School of Medicine and Public Health, University of Newcastle, NSW, Australia

2. Neuroscience Research Australia (NeuRA), Sydney, NSW, Australia

3. Prince of Wales Clinical School, University of New South Wales, Sydney, NSW, Australia

4. Centre for Pain, Health and Lifestyle

5. Research Institute for Primary Care \& Health Sciences, Keele University, Keele, Staffordshire, ST5 5BG, UK

6. The George Institute for Global Health, University of Sydney, Sydney, NSW, Australia

7. Sansom Institute for Health Research, University of South Australia, Adelaide, SA, Australia

8. Hunter New England Population Health, Hunter New England Local health District, NSW Australia

\begin{abstract}
In recent years, there has been increasing interest in studying causal mechanisms in the development and treatment of back pain. The aim of this article is to provide an overview of our current understanding of causal mechanisms in the field. In the first section, we introduce key concepts and terminology. The second section is a brief synopsis of systematic reviews of mechanism studies relevant to the clinical course and treatment of back pain. In the third section, we reflect on the findings of our review to explain how understanding causal mechanisms can inform clinical practice and the implementation of best practice. In the final sections, we introduce contemporary methodological advances, highlight key assumptions of these methods, and discuss future directions to advance the quality of mechanism-related studies in the back pain field.
\end{abstract}

Key words: Back pain, mediation analysis, mechanism, causal inference 


\section{Practice points}

- Understanding causal mechanism in the development of back pain can help identify important targets for treatments.

- Understanding causal mechanisms of treatments can establish how a treatment works, or why it fails. This is important to guide the adaptation of treatments to improve efficacy and facilitate implementation.

- The methodological quality of mechanism studies is poor. Thus, it is difficult to draw firm conclusions about specific mechanisms for back pain treatments.

\section{Research agenda}

- A collective agenda to identify a set of causal mechanisms for common treatments for back pain is needed.

- Over time, we should expect to see a body of evidence emerge - with estimations of mediation effects for common and new treatments.

- These mediation effects could be systematically reviewed and meta-analysed to identify common and specific mechanisms for treatments.

- The combination of rigorously designed and appropriately analysed mechanism-based randomised trials, clear enunciation of causal assumptions, and standardised reporting of mediation effects should lead to stronger quality evidence for underlying causal mechanisms in the clinical course and treatment of back pain. 


\section{Introduction}

Experiencing back pain without knowing what causes it can be highly distressing for the patient. Disappointingly, research so far has failed to provide robust causal explanations to mitigate these uncertainties. Poor understanding of causal factors has stifled the development of effective treatments. This is because treatment targets have only been informed by assumptions or implicit theories, rather than empirical evidence for well-defined causal mechanisms. For the past 16 years, landmark papers in the field have repeatedly highlighted the paucity of evidence on the causes of low back pain. ${ }^{1-3}$ This is despite back pain researchers ranking research into the mechanisms and causes of low back pain as one of the highest research priorities to advance the field. ${ }^{4}$

By understanding causal mechanisms in the clinical course of back pain we can identify important factors that can be targeted in treatment. Although pain and disability are important outcomes for the patient, they are not typical targets of treatments. Treatments are devised to target hypothesised intermediary factors (or mediators) that are causally linked to patient-relevant outcomes such as pain or disability. In other words, treatments generally exert their effects on pain and disability via indirect pathways, which we refer to as 'causal mechanisms'. In a recent mechanism evaluation study, Fordham and colleagues showed that cognitive behavioural therapy (exposure) reduced disability (outcome) via changes in self-efficacy, fear avoidance, and physical functioning (mediators), but not via improvements in mental functioning. ${ }^{5}$ This information gained from a mechanism evaluation is particularly useful in understanding how treatments work, or why they fail.

By understanding causal mechanisms, treatments can be adapted and refined to improve clinical efficacy and to facilitate implementation. Recently, there has been increasing interest in generating evidence for causal mechanisms in the back pain field. ${ }^{6}$ The aim of this article is to provide an overview of current understanding of causal mechanisms in the clinical development and treatment of back pain. The article is divided into five sections:

\section{Key concepts and terminology}

2. Overview of mechanism evaluations in the clinical course and treatment of low back pain

3. Application to clinical practice and implementation

4. Methodological considerations

5. Future directions

\section{Key concepts and terminology}


Many empirical studies in health research aim to establish whether an exposure causes an outcome. ${ }^{7}$ For example, a randomised trial might aim to establish whether an exercise program (exposure) causes less disability (outcome). However, merely establishing a single causal association is often insufficient to explain how or why an exposure causes an outcome. ${ }^{8}$ This approach to understanding causation is often criticised as the 'black-box' approach - because the underlying causal mechanisms are unknown. ${ }^{9,10}$ In the above example, there is no explanation for how an exercise program causes less disability. To overcome this limitation, we can use certain design characteristics and analyses in clinical studies to evaluate causal mechanisms, and help explain how or why a causal association occurs. ${ }^{10}$

A causal mechanism is the pathway that links an exposure (which could be a treatment or observed variation in clinical exposure) to an outcome. ${ }^{9}$ To identify a causal mechanism, a theoretically plausible intermediate variable (often called a mediator) that lies on the causal path between the exposure and outcome needs to be identified. Then, mediating effects (or indirect effects) through that selected mediator can be estimated, often by conducting a form of mediation analysis. ${ }^{10}$

Mediation analysis can quantify causal mechanisms by decomposing the causal effect of the exposure on the outcome (total effect) into indirect and direct effects. The indirect effect is the causal effect of the exposure on the outcome that is channelled through the selected mediator, ${ }^{8,10,11}$ and the direct effect encompasses all other unspecified mechanisms. Quantitatively, the total effect is the sum of the indirect and direct effect, hence the term 'effect decomposition'. By decomposing the total effect into indirect and direct effects, we can gain insight into why an exposure causes an outcome. Importantly, mediation analyses are causal analyses. Thus, it is always necessary to consider the role of confounders in these analyses.

To illustrate the above concepts, the following section outlines examples of the application of mediation analyses to cohort studies and randomised trials. A graphical representation of the above concepts is also shown in Figure 1 with accompanying definitions in Table 1.

(a) Cohort study 


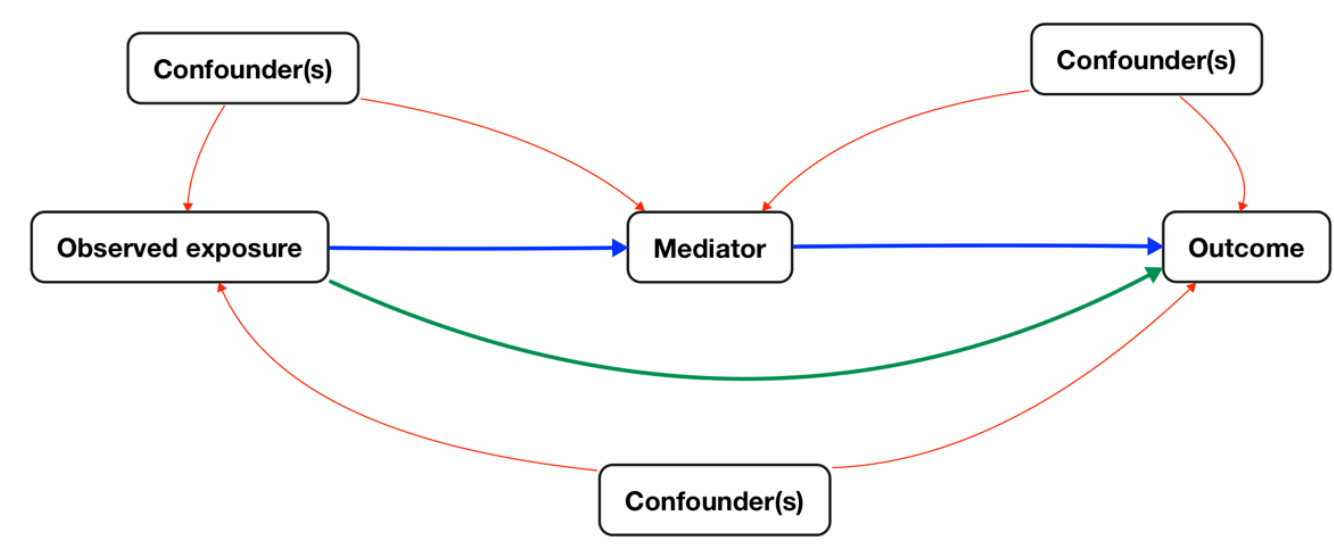

(b) Randomised trial

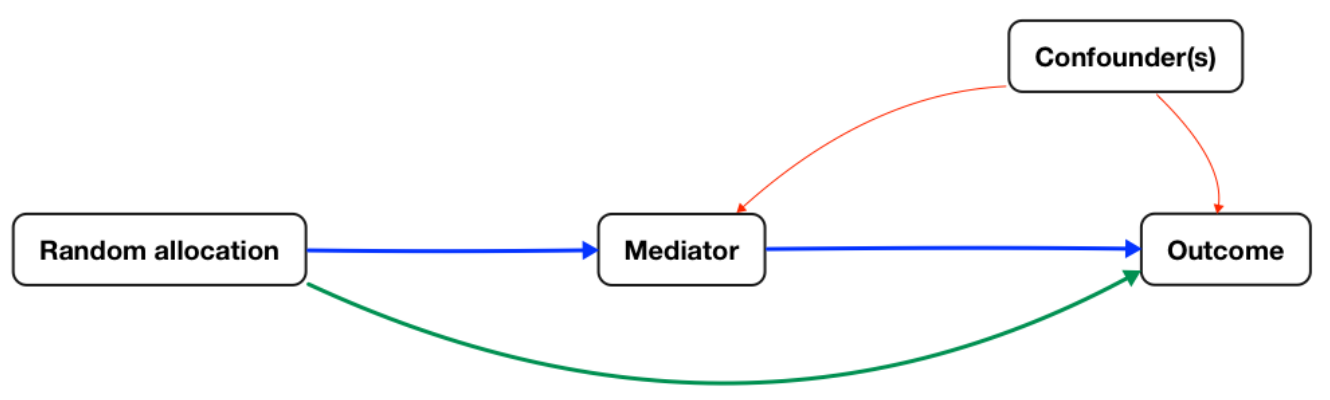

Figure 1. Graphical representation of a single mediator model in a randomised trial and cohort study. Blue arrows represent indirect effects, green arrows represent direct effects, red arrows represent confounding effects.

Table 1. Terms and definitions

\begin{tabular}{|l|l|}
\hline Terms & Definitions \\
\hline Exposure & $\begin{array}{l}\text { A variable that is thought to exert an effect on the outcome; via selected mediators and/or } \\
\text { through a direct causal pathway. In randomised trials, this is often the random allocation of } \\
\text { participants to selected groups (e.g. intervention vs control). In cohort studies, this is often } \\
\text { an observed variable that is not experimentally manipulated (e.g. pain intensity, obesity, } \\
\text { postcode). }\end{array}$ \\
\hline Mediator & $\begin{array}{l}\text { A selected intermediate variable that is thought to channel the causal effect of the exposure } \\
\text { onto the outcome. In other words, a variable that is causally affected by the exposure, which } \\
\text { then causally affects the outcome. }\end{array}$ \\
\hline Outcome & $\begin{array}{l}\text { A key variable that is hypothesised to be influenced by the exposure via a selected mediator, } \\
\text { or any other unspecified mechanism(s). This is often the primary outcome of a randomised } \\
\text { trial or cohort study e.g. pain-related disability. }\end{array}$ \\
\hline Confounder & $\begin{array}{l}\text { A covariate that could induce a spurious (non-causal) apparent association between: } \\
\text { exposure-mediator; exposure-outcome; mediator-outcome. In cohort studies, independent or } \\
\text { mutual sets of confounders may exist for all three effects (indirect, direct, total). In } \\
\text { randomised trials, a set of confounders may only exist for the mediator-outcome effect. }\end{array}$ \\
\hline
\end{tabular}

1.1 Hypothetical examples 
Mediation analyses are often applied to both cohort studies and randomised trials; each addressing different research questions. When mediation analyses are applied to cohort studies, the aim is to identify causal pathways involved in the onset or sequela of a health condition. For example, the transition from acute back pain (exposure) to ongoing disability (outcome) may be mediated via reduced levels of physical activity (mediator). Mechanism evaluations in these instances can support a hypothesised causal mechanism, or perhaps more importantly, identify where the causal mechanism breaks down. For instance, acute pain may reduce physical activity, but that reduction in physical activity may not be the cause of ongoing disability. Quantifying these mechanisms can provide insight into the factors that drive poor (or better) outcome, which can then inform treatment targets (explained in subsequent sections).

When mediation analyses are applied to randomised trials, the aim is to understand why an intervention is, or isn't, effective. ${ }^{11,12}$ For example, an exercise intervention (exposure) may partly exert its effect on disability (outcome) by reducing depressive symptoms (mediator). ${ }^{13}$ Mediation analyses are also particularly useful for explaining why treatments fail. In randomised trials with null findings, a mediation analysis can identify whether it was the intervention that failed to influence the mediator, or whether that mediator failed to influence the outcome, or both. In the above example, the exercise intervention may successfully reduce depressive symptoms, but that shift in depressive symptoms (caused by the intervention) may not cause a reduction in disability. This could be because of an insufficient magnitude of change in depressive symptoms, or simply because depressive symptoms do not cause changes in disability. Understanding where the causal path breaks down by using this approach can be useful to guide the adaptation of treatments (explained in subsequent sections).

\section{2 Key difference between mediation analyses of randomised trials and cohort studies}

From a methodological perspective, the key difference between mechanism evaluations of randomised trials versus cohort studies is how the exposure variable is defined. In randomised trials, the exposure is the random allocation of participants to groups (e.g. exercise vs usual care), whereas in cohort studies, the exposure is usually something that cannot be experimentally manipulated (e.g. smoking vs no smoking). This difference has important repercussions for the internal validity of the mechanism evaluation. ${ }^{14} \mathrm{~A}$ key assumption for accurately identifying causal mechanisms is the "noconfounding' assumption. ${ }^{12,15}$ To meet this assumption, all pathways in the model (Figure 1) should be free from confounding. In a cohort study, despite adjusting for known confounders for all three paths, the assumption may still be unmet because of unknown or unmeasured confounders. ${ }^{16}$ Whereas in a randomised trial (assuming perfect adherence, complete follow-up, and adequate concealment), the treatment-outcome effect, and treatment-mediator effects are free from confounding because randomisation eliminates the effects of known and unknown confounders. However, even in 
randomised trials, the path from the mediator to the outcome could still be confounded, even if known confounders are adjusted for. A major advance in the field was the recent development of the sensitivity analyses, which can provide insight into this impact of the no-confounding assumption. ${ }^{8,17}$

\section{Overview of mechanism evaluations in the clinical course and treatment of low back pain}

\subsection{Causal mechanisms in the clinical course of low back pain}

The clinical course of back pain typically follows a pattern where most patients experience significant improvements in pain and disability in the first six weeks from acute onset. ${ }^{18,19}$ Patients who continue to experience pain beyond six weeks are likely to suffer from significant levels of disability that adds to the burden of back pain. ${ }^{20}$. Mediation analysis can be used to identify the processes that lead to prolonged disability in patients with back pain. For example, mediation analyses of cohort studies have tested whether psychological variables underlie the development of prolonged disability. ${ }^{21,22}$ These studies were systematically reviewed and meta-analysed. ${ }^{23}$ From 12 studies that reported a total of 36 mediator models, reduced distress, reduced fear, and increased self-efficacy were shown to mediate the pain-disability relationship. Contrary to prevailing view, ${ }^{24,25}$ decreased catastrophization was not a mediator. ${ }^{23}$ Interestingly, the identified psychological mechanisms only explained a moderate proportion of the total effect; approximately between 20 to $33 \%$. This means that there are likely to be unexplained causal mechanisms that have yet to be identified (i.e. remaining direct effects accounted for a significant proportion of the total effect - 67 to 80\%). Perhaps these unexplained mechanisms are biological, such as neuro-inflammatory, immunological ${ }^{26}$ and endocrine systems ${ }^{27}$ that upregulate nociception. Establishing the causal pathways that link these constructs will provide more comprehensive explanations for why back pain causes disability.

\subsection{Causal mechanisms in treating low back pain}

In 2013, Mansell et al. ${ }^{6}$ systematically reviewed mediators of psychological interventions that aimed to reduce disability in patients with musculoskeletal conditions. Their review found that increased self-efficacy, reduced pain catastrophizing, accurate pain beliefs, increased pain coping, and increased psychological flexibility were important mediators. Since the publication of this review, more studies $^{5,28-31}$ have investigated causal mechanisms of various interventions for back pain. These studies along with those reviewed by Mansell et al. are summarised in Table 2.

Table 2. Causal mechanism evaluations of treatments for back pain

\begin{tabular}{llll}
\hline Study & Intervention (exposure) & Mediators tested & Outcome \\
\hline
\end{tabular}




\begin{tabular}{ll}
\hline Focht et al 2005 & $\begin{array}{l}\text { Exercise }(n=80) \\
\text { Dietary weight loss }(n=82) \\
\text { Combination therapy }(n=76) \\
\text { Attention control }(n=78)\end{array}$ \\
Nicassio et al 1997 & $\begin{array}{l}\text { Behavioural therapy }(n=48) \\
\text { Control: Education }(n=38)\end{array}$ \\
Leeuw et al 2008 & $\begin{array}{l}\text { Graded exposure in vivo }(n=42) \\
\text { Graded activity }(n=43)\end{array}$ \\
Robinson et al 2013 & $\begin{array}{l}\text { Exposure therapy }(n=70) \\
\text { Information booklet }(n=57)\end{array}$ \\
Seymour et al 2009 & $\begin{array}{l}\text { Exercise therapy delivered by } \\
\text { physiotherapists } \\
\text { Exercise therapy delivered by certified } \\
\text { exercise instructors }\end{array}$
\end{tabular}

Smeets et al $2006 \quad$ Cognitive-behavioural therapy $(n=55)$ Active physical therapy $(n=52)$ Combination therapy $(n=55)$

Waiting list $(n=49)$

Spinhoven et al 2004 Operant behavioural therapy with cognitive coping skills training $(n=59)$ Operant behavioural therapy with group discussion $(n=58)$ Waiting list $(n=31)$

Turner et al 2007

Wicksell et al 2010

Fordham et al. $2017^{5}$

Advice plus Cognitive Behavioural Intervention $(n=468)$

Advice alone $(n=233)$

Mansell, et al. $2016^{28} \quad$ Reactivation intervention $(n=119)$ Usual care $(n=121)$
- Stair-climbing self-efficacy

- Walking self-efficacy

- Helplessness

- Pain coping

- Social support

- Pain catastrophizing

- Perceived harmfulness of activities

- Fear of specific neck movements

- Exercise adherence self-efficacy (barriers adherence, time adherence)

- Self-management self-efficacy (exercise, pain management, symptom management)

- Attendance

- Exercise maintenance

- Pain catastrophizing

- Internal pain control

- Catastrophizing

- Pain coping

- Internal pain control

- External pain control

- Self-efficacy

- Pain beliefs

- Catastrophizing

- Coping (relaxation)

- Psychological flexibility

- Self-efficacy

- Pain intensity

- Anxiety

- Depression

- Fear-avoidance

- Pain self-efficacy

- Fear-avoidance

- Physical functioning

- Mental functioning

- Fear-avoidance beliefs

- Pain catastrophizing
Mobility (walking and stair-climbing) and Pain

Disability, Depression, Pain, Myalgia

Functional disability, Main complaints

Neck disability

Function (muscle strength, exercise capacity, physical function), Pain

Disability, Pain, Depression, Patientspecific complaints

Activity tolerance, Pain (intensity and behaviour),

Depression

Jaw use limitation, Pain (intensity and activity interference)

Pain disability, Life satisfaction

Disability

Disability

Pain intensity, Pain bothersomeness, Disability 


\begin{tabular}{|c|c|c|c|}
\hline Whittle et al. $2016^{30}$ & $\begin{array}{l}\text { Minimal intervention strategy }(n=143) \\
\text { Usual GP care }(n=171)\end{array}$ & $\begin{array}{l}\text { - } \quad \text { Pain catastrophizing } \\
\text { - } \text { Fear-avoidance beliefs } \\
\text { - } \text { Distress } \\
\text { increase exercise, stay active and } \\
\text { moving) }\end{array}$ & Disability \\
\hline Tetsunaga et al. 2016 & $\begin{array}{l}\text { Tramadol-acetaminophen }(n=36) \\
\text { Celecoxib }(n=37)\end{array}$ & - Pain relief & Apathy \\
\hline Mansell et al. $2016^{32}$ & $\begin{array}{l}\text { Stratified care }(n=93) \\
\text { Current best care }(n=45)\end{array}$ & $\begin{array}{l}\text { Distress (as a latent variable } \\
\text { represented by fear-avoidance, } \\
\text { pain catastrophizing, anxiety and } \\
\text { depression) }\end{array}$ & $\begin{array}{l}\text { Disability } \\
\text { Four-month follow- } \\
\text { up }\end{array}$ \\
\hline & & - Pain intensity & \\
\hline
\end{tabular}

Mediators highlighted in bold text were significant mediators identified.

In addition to the studies reviewed by Mansell et al., ${ }^{6}$ six additional studies examined mechanisms of interventions for back pain. Interestingly, similar mediators as those found by Mansell et al. were identified for psychological interventions, including pain self-efficacy, fear-avoidance, pain catastrophizing, and distress. Notwithstanding limitations, this summary supports a theory of shared mechanisms. ${ }^{33}$ That is, apparently different psychological interventions may produce similar effects on outcome via a common set of mediators. For example, cognitive behavioural therapy, mindfulnessbased therapies, and acceptance and commitment therapy may all exert their effects on outcome via a common set of psychological mediators (e.g. increased self-efficacy, reduced catastrophizing).

Separate to psychological interventions, two studies investigated causal mechanisms for Tai Chi, ${ }^{29}$ and Tramadol-acetaminophen. ${ }^{31}$ It is difficult to draw conclusions from these single studies, until further replication. There is also further work being conducted to evaluate the mechanisms for pain education ${ }^{34}$ and lifestyle-based interventions ${ }^{13}$ for back pain.

Although the evidence for treatment mechanisms is growing, it is still difficult to draw firm conclusions about specific mechanisms for back pain treatments. Confidence in the evidence is limited by the small number of studies for each type of intervention, and specific models with consistent mediators and outcomes. There is more work to be done in this area. A collective agenda to identify a set of causal mechanisms for common treatments for back pain is needed.

\section{Application to clinical practice and implementation}

In clinical practice, it is useful to know which treatment targets will lead to improvements in patientrelated outcomes. By knowing these targets, clinicians can specifically tailor their treatments to selectively target appropriate mechanisms. The systematic review of cohort studies shows that psychological mechanisms including: increased self-efficacy, reduced fear, reduced distress can serve 
as treatment targets. As shown by Mansell et al, and more recent mechanism evaluations of randomised trials, increased self-efficacy, reduced fear, reduced catastrophizing, reduced distress, and increased psychological flexibility were shown to mediate treatment outcomes. For clinicians, it would be important to include strategies and techniques which have been shown to specifically influence these known mediators. For example, to target self-efficacy, Bandura's techniques of modelling, mastery, and verbal persuasion ${ }^{35,36}$ could be incorporated into psychologically-informed interventions.

It is also important for clinicians to consider harmful mechanisms that could carry an adverse effect of an exposure onto the outcome. ${ }^{37}$ Such harmful mechanisms could be driven by unnecessary management, for example, spinal imaging (exposure) may increase the patient's belief that pain is a sign of tissue damage (mediator), which could lead to increased disability (outcome). Harmful mechanisms could also be inadvertently triggered by unintended consequences of an effective intervention. For example, a group based exercise therapy (exposure) may exert its effects through positive changes in physical wellbeing (positive mediator), but incompatible social interactions between participants could increase distress (negative mediator) that may suppress positive effects and eventually attenuate the overall treatment effect on the outcome (disability). These mechanisms have not been tested, but are plausible hypotheses that should feature in clinical reasoning.

In clinical practice, evidence-based interventions are frequently adapted in the process of implementation. ${ }^{38}$ Adaptations typically occur because of resource constraints or to meet the needs of different population groups. For organisational and policy decisions, mechanism evaluations of complex interventions can provide an empirical basis to identity 'core' and 'non-core' intervention components to guide policy decisions regarding the necessary intervention components for effective implementation. Specifically, intervention components contributing to 'core' mediating pathways should be retained and emphasis placed on fidelity of their implementation. Intervention components directed towards 'non-core' factors that are not associated with a change in outcome potentially could be removed without compromising the efficacy of the intervention. In doing so, the intervention may be perceived as less complex, be more efficiently implemented and potentially more cost effective. There are also causal mechanisms that could inform how implementation interventions work. That is, the process of implementation itself, not the mechanisms of the intervention/program being implemented. This area is gaining attention, ${ }^{39}$ but is beyond the scope of this paper.

\section{Methodological considerations}


Both systematic reviews of mediation studies in the field, ${ }^{6,23}$ and subsequent work, ${ }^{40,41}$ have noted that most mechanism studies are of poor methodological quality. Of 36 mediation models in cohort designs, $59 \%$ used cross-sectional designs that violate the assumption of temporal precedence necessary for causal inference. ${ }^{23}$ To overcome this problem, longitudinal studies should include at least 3 waves of measurement points to determine the temporal sequence of the exposure, mediator, and outcome. Testing mediation models in cross-sectional designs should be discouraged, unless a unidirectional temporal sequence can be supported with strong theoretical rationale.

Mediation analyses are causal analyses and so should consider the effects of known and unknown confounders. ${ }^{42}$ The 2015 systematic review of cohort studies identified that only $25 \%$ of mediator models adjusted for known confounders, and none conducted sensitivity analyses to assess the impact of unknown confounders. ${ }^{23}$ Confounding is also important for randomised designs because the mediator cannot be randomised. Among those studies listed in Table 2, 53\% accounted for known confounders of the mediator-outcome path, but none conducted sensitivity analyses to assess the impact of unknown confounders. This is a critical limitation to causal inference and it is encouraged that future studies identify and adjust for a set of possible confounders when conducting mediation studies. This should also be followed up with a sensitivity analysis to assess how hypothesised levels of unknown confounding would invalidate estimated indirect effects. ${ }^{43,44}$

Recent advances from the causal inference literature have also provided nuanced definitions of indirect, direct and total effects that are grounded in the counterfactual/potential-outcomes framework. ${ }^{10}$ These methods are offer advantages to older methods; they have wide applicability to accommodate non-linear models (e.g. binary outcomes such as recovery), and are based on clear assumptions for making causal inferences (e.g. no confounding assumption). ${ }^{8,43}$ These frameworks have also been extended to account for interactions between the exposure and mediator - in cases where the effect of the mediator on outcome is dependent on the treatment received (e.g. the effect of a mediator such as depressive symptoms on disability might differ for patients who received an exercise intervention versus those who did not). ${ }^{45}$ The framework also handles multiple intertwined mediators - when there are more than one putative mediators that are causally linked (e.g. selfefficacy and depressive symptoms might be mediators, but increasing self-efficacy could reduce depressive symptoms). ${ }^{46}$ Future work should apply and disseminate these contemporary methods for mediation analyses.

\section{Future directions}


Effective treatment of low back pain is often stifled by limited understanding of targets that are based on implicit theories about their causal mechanisms. Future research should describe and test these implicit theories for common treatments. ${ }^{6,30,47}$ To do so, a comprehensive program of work would be required. This might begin with qualitative evaluations and cohort studies to generate new theories for biologically plausible treatment targets. This could be followed by a priori planned mechanism-based randomised trials to test plausible mechanisms of treatments. An alternative approach might be to adapt existing treatments by understanding their underlying mechanisms. This could be done by leveraging data from existing trials. In these cases, it would be important to specify a priori analysis protocols. As a general consideration, the analysis should be theoretically driven; mediators should be measured at time-points at which they're expected to change; potential baseline and postrandomisation confounders should be collected; and sensitivity analyses should be conducted to assess the effects of unknown confounding. Undertaking this work is feasible (with the right expertise and planning), often without substantial additional resources, and can provide important information for clinical practice, research and implementation.

Over time, we should expect to see a body of evidence emerge - with estimations of mediation effects for common and new treatments. These mediation effects could be systematically reviewed and metaanalysed to identify common and specific mechanisms for treatments. However, for such reviews to accurately assess heterogeneity and risk of bias - development of a specific reporting guideline will be crucial ${ }^{48}$ (now under development). ${ }^{49}$ The combination of rigorously designed and well conducted mechanism-based randomised trials, clear enunciation of causal assumptions, and standardised reporting of mediation effects should lead to stronger quality evidence for underlying causal mechanisms in the clinical course and treatment of back pain.

Conflicts of interest: None declared. 


\section{References}

1 Maher C, Underwood M, Buchbinder R. Non-specific low back pain. Lancet 2016; 6736: 1-12.

2 Hoy D, Brooks P, Blyth F, Buchbinder R. The Epidemiology of low back pain. Best Pract Res Clin Rheumatol 2010; 24: 769-81.

3 Deyo RA, Weinstein JN. Low back pain. $N$ Engl J Med 2001; 344: 363-70.

4 Costa LDCM, Koes BW, Pransky G, Borkan J, Maher CG, Smeets RJEM. Primary care research priorities in low back pain: an update. Spine (Phila Pa 1976) 2013; 38: 148-56.

$5 \quad$ Fordham B, Ji C, Hansen Z, Lall R, Lamb SE. Explaining How Cognitive Behavioural Approaches Work for Low Back Pain. Spine (Phila Pa 1976) 2017; : 1.

6 Mansell G, Kamper SJ, Kent P. Why and how back pain interventions work: What can we do to find out? Best Pract Res Clin Rheumatol 2013; 27: 685-97.

7 Hernan MA. A definition of causal effect for epidemiological research. $J$ Epidemiol Community Heal 2004; 58: 265-71.

8 Imai K, Keele L, Tingley D. A general approach to causal mediation analysis. Psychol Methods 2010; 15: 309-34.

9 Imai K, Keele L, Tingley D, Yamamoto T. Unpacking the Black Box of Causality: Learning about Causal Mechanisms from Experimental and Observational Studies. Am Polit Sci Rev 2011; 105: 765-89.

10 VanderWeele T. Explanation in causal inference: methods for mediation and interaction. Oxford University Press, 2015.

11 Emsley R, Dunn G, White IR. Mediation and moderation of treatment effects in randomised controlled trials of complex interventions. Stat Methods Med Res 2010; 19: 237-70.

12 Dunn G, Emsley R, Liu H, et al. Evaluation and validation of social and psychological markers in randomised trials of complex interventions in mental health. NIHR Rep 2015; 19. DOI:10.3310/hta19930.

13 Lee H, Wiggers J, Kamper SJ, et al. Mechanism evaluation of a lifestyle behavioural intervention for patients with musculoskeletal pain who are overweight or obese. Protocol for a causal mediation analysis. BMJ Open 2017; Accepted.

14 Agoritsas T, Merglen A, Shah ND, O 'donnell M, Guyatt GH. Adjusted Analyses in Studies Addressing Therapy and Harm Users' Guides to the Medical Literature Clinical Scenario. JAMA 2017. DOI:10.1001/jama.2016.20029.

15 Imai K, Keele L, Yamamoto T. Identification, Inference and Sensitivity Analysis for Causal Mediation Effects. Stat Sci 2010; 25: 51-71.

16 Goodman SN, Schneeweiss S, Baiocchi M. Using Design Thinking to Differentiate Useful From Misleading Evidence in Observational Research. Jama 2017; 317: 705-7.

17 Albert JM, Wang W. Sensitivity analyses for parametric causal mediation effect estimation. Biostatistics 2014; 16: 339-51. 
18 van Tulder M, Becker A, Bekkering T, et al. Chapter 3. European guidelines for the management of acute nonspecific low back pain in primary care. Eur Spine J 2006; 15 Suppl 2: S169-91.

19 Henschke N, Maher CG, Refshauge KM, et al. Prognosis in patients with recent onset low back pain in Australian primary care: inception cohort study. BMJ 2008; 337: a171. Costa LDCM, Maher CG, Hancock MJ, McAuley JH, Herbert RD. The prognosis of acute and persistent low-back pain: a meta analysis. Can Med Assoc J 2012; 184: 1229-30.

21 Nieto R, Miró J, Huguet A. Pain-related fear of movement and catastrophizing in whiplashassociated disorders. Rehabil Psychol 2013; 58: 361-8.

22 Costa LDCM, Maher CG, McAuley JH, Hancock MJ, Smeets RJEM. Self-efficacy is more important than fear of movement in mediating the relationship between pain and disability in chronic low back pain. Eur J Pain 2011; 15: 213-9.

23 Lee H, Hübscher M, Moseley GL, et al. How does pain lead to disability? A systematic review and meta-analysis of mediation studies in people with back and neck pain. Pain 2015; 156: 988-97.

24 Quartana PJ, Campbell CM, Edwards RR. Pain catastrophizing: a critical review. Expert Rev Neurother 2009; 9: 745-58.

25 Smeets RJEM, Vlaeyen JWS, Kester ADM, Knottnerus JA. Reduction of pain catastrophizing mediates the outcome of both physical and cognitive-behavioral treatment in chronic low back pain. J Pain 2006; 7: 261-71.

26 Austin PJ, Moalem-Taylor G. The neuro-immune balance in neuropathic pain: involvement of inflammatory immune cells, immune-like glial cells and cytokines. $J$ Neuroimmunol 2010; 229: $26-50$.

27 Chapman CR, Tuckett RP, Song CW. Pain and stress in a systems perspective: reciprocal neural, endocrine, and immune interactions. J Pain 2008; 9: 122-45.

28 Mansell G, Hill JC, Main C, Von Korff M, van der Windt D. Mediators of Treatment Effect in the Back In Action Trial. 2016 DOI:10.1097/AJP.0000000000000463.

29 Hall AM, Kamper SJ, Emsley R, Maher CG. Does pain-catastrophising mediate the effect of tai chi on treatment outcomes for people with low back pain? Complement Ther Med 2016; 25: $61-6$.

30 Whittle R, Mansell G, Jellema P, van der Windt D. Applying causal mediation methods to clinical trial data: What can we learn about why our interventions (don't) work? Eur J Pain 2016; InPress.

31 Tetsunaga T, Tetsunaga T, Tanaka M, Nishida K, Takei Y, Ozaki T. Effect of tramadol/acetaminophen on motivation in patients with chronic low back pain. Pain Res Manag 2016; 2016. DOI:10.1155/2016/7458534.

32 Mansell G, Hill JC, Main C, Vowles KE, van der Windt D. Exploring What Factors Mediate 
Treatment Effect: Example of the STarT Back Study High-Risk Intervention. J Pain 2016; 17: $1237-45$.

33 Burns JW. Mechanisms, mechanisms, mechanisms : it really does all boil down to mechanisms. 2016; 157: 2393-4.

34 Lee H, Moseley GL, Hübscher M, et al. Understanding how pain education causes changes in pain and disability: protocol for a causal mediation analysis of the PREVENT trial. $J$ Physiother 2015; 61: 156.

35 Bandura A. Self-efficacy: toward a unifying theory of behavioral change. Psychol Rev 1977; 84: 191.

36 Williams DM. Outcome expectancy and self-efficacy: theoretical implications of an unresolved contradiction. Pers Soc Psychol Rev 2010; 14: 417-25.

37 Bonell C, Jamal F, Melendez-Torres GJ, Cummins S. 'Dark logic': theorising the harmful consequences of public health interventions. J Epidemiol Community Health 2015; 69: 95-8.

38 Stirman SW, Miller CJ, Toder K, Calloway A. Development of a framework and coding system for modifications and adaptations of evidence-based interventions. Implement Sci 2013; 8: 65.

39 Anselmi L, Binyaruka P, Borghi J. Understanding causal pathways within health systems policy evaluation through mediation analysis: an application to payment for performance (P4P) in Tanzania. Implement Sci 2017; : 1-18.

40 Lee H, Hübscher M, McAuley JH. Importance of quantifying indirect effects from mediation analyses. Pain 2015; 156: 2634-7.

41 Lee H, Hübscher M, McAuley JH. The Implications of Using Binary Outcomes in Mediation Analysis. J Pain 2016; 17: 1045-6.

42 MacKinnon DP, Fairchild AJ, Fritz MS. Mediation analysis. Annu Rev Psychol 2007; 58: 593614.

43 Keele L. Causal Mediation Analysis: Warning! Assumptions Ahead. Am J Eval 2015; 36: $500-13$.

44 Imai K, Yamamoto T. Identification and Sensitivity Analysis for Multiple Causal Mechanisms: Revisiting Evidence from Framing Experiments. Polit Anal 2013; 21: 141-71.

45 Valeri L, Vanderweele TJ. Mediation analysis allowing for exposure-mediator interactions and causal interpretation: theoretical assumptions and implementation with SAS and SPSS macros. Psychol Methods 2013; 18: 137-50.

46 Daniel RM, De Stavola BL, Cousens SN, Vansteelandt S. Causal mediation analysis with multiple mediators. Biometrics 2015; 71: 1-14.

47 Moore GF, Audrey S, Barker M, et al. Process evaluation of complex interventions: Medical Research Council guidance. BMJ 2015; 350: h1258-h1258.

48 Liu S-H, Ulbricht CM, Chrysanthopoulou SA, Lapane KL. Implementation and reporting of 
causal mediation analysis in 2015: a systematic review in epidemiological studies. BMC Res Notes 2016; 9: 354.

49 Lee H, Kamper SJ, Hübscher M, et al. AGREMA: a guideline for reporting mechanism evaluations in randomised controlled trials. EQUATOR Netw Website. 2016.

http://www.equator-network.org/wp-content/uploads/2009/02/LEE-Brief-abstract-forEQUATOR.pdf (accessed Nov 21, 2016). 